\title{
Féeries
}

Études sur le conte merveilleux, XVII ${ }^{\mathrm{e}} \mathrm{XIX}{ }^{\mathrm{e}}$ siècle

\section{La « dramatisation » de Tanzaï et Néadarné par Charles Collé}

Une caricature involontaire du conte

\section{Régine Jomand-Baudry}

\section{(2) OpenEdition}

\section{Journals}

Édition électronique

URL : http://journals.openedition.org/feeries/363

DOI : $10.4000 /$ feeries.363

ISSN : 1957-7753

\section{Éditeur}

UGA Éditions/Université Grenoble Alpes

\section{Édition imprimée}

Date de publication : 1 octobre 2007

Pagination : 155-174

ISBN : 978-2-84310-101-4

ISSN : 1766-2842

\section{Référence électronique}

Régine Jomand-Baudry, «La « dramatisation » de Tanzaï et Néadarné par Charles Collé », Féeries [En ligne], 4 | 2007, mis en ligne le 31 octobre 2008, consulté le 07 septembre 2020. URL : http:// journals.openedition.org/feeries/363; DOI : https://doi.org/10.4000/feeries.363

Ce document a été généré automatiquement le 7 septembre 2020.

(c) Féeries 


\title{
La « dramatisation » de Tanzaï et Néadarné par Charles Collé
}

\author{
Une caricature involontaire du conte
}

\author{
Régine Jomand-Baudry
}

1 L'AUDACIEUX AUTEUR DE TANZAÏ ET NÉADARNÉ, conte licencieux et parodique qui fit date dans l'histoire du genre, nourrissait une véritable passion pour le théâtre. Spectateur à titre gratuit de la Comédie-Française dès $1726^{1}$, discret collaborateur de Romagnési avec François Riccoboni et Pierre Biancolelli (Dominique) pour plusieurs comédies et particulièrement pour les couplets des parodies d'opéras, comme le signale Palissot, et pour celles du répertoire du Théâtre-Français à partir de $1729^{2}$, il a aussi ponctuellement chaussé le cothurne dans plusieurs théâtres de société ${ }^{3}$, ce qui est moins connu. Si sa collaboration au Théâtre-Italien fut sans doute pour lui une école pour l'apprentissage des subtilités du dialogue et de l'esprit de la parodie, comme le souligne Jean Sgard ${ }^{4}$, on peut s'étonner que cet attrait pour la scène ne se soit pas matérialisé dans son œuvre officielle : car même si ses deux dialogues, La Nuit et le moment et Le Hasard du coin du feu se rapprochent de l'écriture théâtrale par leur forme et ont souvent été représentés à l'époque moderne, ils n'ont pas été conçus pour la scène et conservent délibérément un point de vue narratif ${ }^{5}$.

2 Ses contes n'ont cependant pas manqué d'être étroitement associés ou encore adaptés à la scène dès le XVIII ${ }^{e}$ siècle, l'activité privilégiée de l'auteur à l'intersection des deux champs théâtral et littéraire favorisant de tels transferts. On relève alors deux types de rapports entre les contes et la scène.

3 Le premier type de relation n'engage pas une intertextualité précise entre œuvre narrative et œuvre scénique, il relève d'une stratégie commerciale dans laquelle l'auteur du conte a pu être partie prenante. Il s'agit de focaliser l'intérêt du public à un moment donné, moins sur un texte précis que sur un sujet, en le promouvant simultanément sur la scène et dans le circuit spécifiquement littéraire, afin de créer un effet de synergie : c'est là une manière de construire artificiellement la mode. Il ne fait aucun doute que Le Sylphe ait bénéficié d'une telle stratégie du succès ${ }^{6}$ grâce à la création de La Sylphide de Dominique et Romagnési à la Comédie-Italienne au moment 
même de la publication de ce tout premier texte de Crébillon. On peut comparer ce cas de figure, qui ressemble à une opération de lancement, à celui de Tanzaï et Néadarné. En 1735, au moment où sont données deux nouvelles éditions du conte, les Comédiens Italiens proposent une pièce intitulée Le Conte de fées ou des fées signée Romagnési et Riccoboni, dont le scénario n'est pas sans rapport avec le Tanzaï de Crébillon, comme le soulignent les Anecdotes dramatiques ${ }^{7}$ de Clément et Laporte. Il s'agit alors de tirer profit de sa célébrité (même de scandale), en le prolongeant indirectement sur la scène. Même si chaque œuvre demeure distincte par son titre, les parentés sont aisément reconnaissables et suffisent à susciter l'intérêt du public. On trouve dans le répertoire de la Comédie-Italienne, à condition d'établir le lien entre l'actualité narrative et théâtrale, nombre de ces interactions non explicitement affichées. L'œuvre scénique, loin d'être inféodée à un hypotexte, joue plutôt sur des allusions ou des correspondances scénariques, des références, des annonces de transgénéricité, la comédie s'emparant ici du conte de fée.

4 À côté de cette stratégie commerciale fréquente dans un théâtre institutionnalisé comme la Comédie-Italienne, l'adaptation pour la scène d'un conte précis relève d'une autre démarche, qui, si elle peut ne pas être étrangère à la mode, s'exerce avec des intentions différentes vis-à-vis du texte source qui n'est plus seulement un prétexte. Nous centrerons notre étude sur un exemple curieux qui est la transposition pour la scène de Tanzaï et Néadarné par Collé pour le théâtre de société du duc d'Orléans. Dans cette pièce qui n'a finalement jamais été jouée, mais que Collé a publiée dans son Théâtre de société ${ }^{8}$, l'auteur opte, dans le but de rester fidèle au ton du conte, pour le genre de la tragi-comédie. Or, si ce choix est justifiable, il débouche cependant sur une pièce caricaturale. Cette expérience d'écriture que Collé commente lui-même comme un fiasco, pose le problème de la « théâtralité du sujet » du conte, et peut-être de tout conte parodique et libertin.

De la prédisposition du conte à la dramatisation

5 Tanzaï et Néadarné ne semble avoir été adapté pour le théâtre qu'à deux reprises au cours du XVIII ${ }^{\mathrm{e}}$ siècle. Seule la scène privée s'est autorisée, peu après sa publication, la reprise de ce conte sulfureux par lequel le scandale était arrivé. Selon L'Observateur, ouvrage polygraphique et périodique ${ }^{9}$, une farce représentant l'histoire de l'Écumoire « au naturel » fut jouée en février 1735 dans une maison particulière du Faubourg SaintAntoine lors du Carnaval. Mais le journaliste ne donne pas davantage d'informations sur cette adaptation sans doute audacieuse, et vraisemblablement réservée à un très petit cercle de spectateurs choisis. Collé a-t-il eu connaissance de cette première " dramatisation " du conte ? Toujours est-il que plusieurs années plus tard, en 1754, l'ami de longue date de Crébillon

- celui qui sera son exécuteur testamentaire -, entreprend une tragi-comédie en un acte et en vers, destinée au théâtre de société du duc d'Orléans. La date d'écriture de la pièce ${ }^{10}$, fort éloignée de celle de la publication de son texte source dont elle revendique la filiation par l'identité du titre, n'est cependant pas anodine : elle correspond à la période de retour de Crébillon sur la scène littéraire après douze années de silence éditorial : malgré le succès très mitigé des Heureux Orphelins et peu avant la publication d'Ah quel conte ! l'auteur de Tanzaï est à nouveau un centre d'intérêt pour l'élite cultivée du temps. Son œuvre précédente est loin d'être oubliée et particulièrement le conte de Tanzaï qui a connu au moins deux rééditions récentes en 1749 et en 1751, ce qui indique la pérennité de son succès. 
6 Profiter de l'engouement qui a cours autour du nom de Crébillon et s'inscrire dans le mouvement de la mode ? Rendre hommage à un ami et contribuer à sa renommée en réactualisant un conte dont le caractère provocateur est désormais désamorcé ? Faire sa cour au Duc qui connaissait Crébillon de longue date au point de le secourir dans son infortune en 1753 et pour le théâtre de qui Collé est alors le fournisseur attitré ? Les motivations ne manquent pas pour expliquer le choix de ce sujet pour une pièce qui comme l'affiche son titre entend bien être une adaptation fidèle de ce conte à l'extravagance provocatrice.

7 Il n'est pas inutile de rappeler en guise d'ouverture, les spécificités de la transmodalisation du narratif au dramatique, pour employer le vocabulaire de Genette $^{11}$, lorsqu'elle s'exerce à partir du conte. Elle ouvre sur deux questions principales : en quoi le genre du conte est-il prédisposé à passer à la scène ? Sa transmutation au théâtre suppose-t-elle un genre d'arrivée privilégié ?

Le conte dans sa forme canonique apparaît comme le genre narratif le mieux adapté à la scène tout d'abord par la linéarité de sa fable et par le schématisme qui caractérise sa structure, ses grandes articulations étant suffisamment nettes pour que soit facilité un découpage scénarique en séquences transposables en actes et en scènes. D'autres éléments plaident aussi en faveur de ce type de transmodalisation qui, comme l'histoire littéraire en témoigne, a très massivement lieu dans le sens du narratif vers le théâtre plutôt que l'inverse : mieux que la complexité événementielle du roman et son traitement souple de la temporalité, la brièveté du conte et l'ordre de ses événements s'accordent avec les nécessités de la représentation scénique ; à côté de ces facteurs macrostructurels, le conte recèle une forme de spectaculaire liée au merveilleux, particulièrement goûtée du public du théâtre et de l'opéra au XVIII ${ }^{e}$ siècle, où par l'art des machines sont mises en représentation sous les yeux des spectateurs les illusions du surnaturel. Représenter ce qui n'existe pas, rendre visible ce que l'écrit offre à la seule imagination, est l'une des fonctions propres au théâtre auquel le conte peut fournir un réservoir de scènes inventives qui seront autant de défis pour les machinistes.

9 En revanche, certaines caractéristiques du conte offrent moins de facilités : comment mettre en scène des personnages exclusivement par la parole, sur le mode mimétique, alors qu'ils ne sont présentés que de l'extérieur par le narrateur et alors qu'ils sont la plupart du temps dénués de toute intériorité ? Comment faire parler ce qui n'est pour reprendre la terminologie de Propp, qu'une fonction?

Or, l'invention du conte à la manière de Crébillon résout partiellement un certain nombre de ces difficultés tout en en ajoutant d'autres, comme celle de la représentation du libertinage. En effet, comme le rappelle Catherine Ramond ${ }^{12}$, la veine du conte oriental, parodique et libertin que Tanzaï inaugure en 1734, dans son mouvement de subversion du modèle antérieur, opère un rapprochement notable à l'égard du texte de théâtre sur certains points alors qu'il tend à s'en éloigner sur d'autres.

11 Si la complexification structurelle et son éclatement s'avèrent plutôt un obstacle, l'abondance du dialogue à tous les niveaux, soit qu'il brouille par le recours à la polyphonie énonciative ou par "la prolifération du discours métatextuel ", le statut surplombant du narrateur, soit qu'il s'inscrive directement dans de fréquentes scènes d'échange entre les personnages, constitue plutôt une mutation favorable à la transposition scénique. 
«L'intrusion du spectacle dans sa forme transgressive, le voyeurisme ${ }^{13}$ », et surtout le caractère systématiquement sexuel des scènes de dénouements, s'ils répondent aux nécessités de la visualisation théâtrale, comme le souligne Catherine Ramond, rencontrent néanmoins l'obstacle de la censure morale : ce que l'on peut lire « en toute innocence " grâce au langage gazé, ne supporte guère la représentation directe, à moins que l'on n'écrive pour des scènes très privées spécialisées dans le théâtre érotique ${ }^{14}$.

Mais à quel genre théâtral le conte correspond-il le mieux ? L'histoire littéraire nous enseigne que c'est le plus fréquemment dans la comédie ${ }^{15}$ sous toutes ses formes, avec ou sans divertissements, et notamment dans l'opéra-comique, que le conte est adapté, sa fin heureuse désignant logiquement cet homologue théâtral. L'étude conduite par Catherine Ramond met au jour un autre motif qui peut servir à expliquer l'élection du genre d'arrivée : par sa finalité morale, le conte entend bien prendre position sur des faits de l'actualité politique et sociale tout autant qu'analyser les conduites. Par là, « il prend explicitement le relais de la comédie ${ }^{16}$ ", et il est donc tout naturel qu'il s'incarne dans ce genre. Un dernier aspect du conte parodique explique cette prédétermination pour la comédie : son ton léger de divertissement, qui s'il n'évite pas le comique burlesque ou même gaulois, joue sur la distance parodique et l'humour. Le conte est donc a priori assez spontanément associé au divertissement comique.

4 Ces indicateurs d'ordre général doivent être modulés en fonction de données extérieures au texte source, mais dont l'influence sur l'orientation de sa transposition est déterminante : il s'agit du type de théâtre de destination et des intentions de l'adaptateur vis-à-vis de son texte de base. S'agit-il pour lui d'un simple prétexte à partir duquel il pourra inventer à sa guise, veut-il jouer de l'intertextualité sur le mode parodique ou au contraire rester fidèle à l'esprit de sa source ? Quant au théâtre de destination, que ce soit la scène des Italiens, le théâtre de la Foire ou encore le théâtre de société - dont le degré de liberté varie selon les scènes -, chaque scène impose non seulement un ton, mais une forme, celle de la comédie à divertissements ou de l'opéracomique par exemple, avec une latitude plus ou moins grande avec laquelle le dramaturge devra compter.

5 Sur ce paysage brossé à grands traits comment se présente le cas particulier de l'adaptation de Tanzaï par Collé ? Comment le dramaturge intègre-t-il les contraintes propres à la transposition scénique, en tenant compte des exigences du théâtre de destination? Quel rapport institue-t-il avec son texte source? Nous verrons que malgré la concentration dramatique de l'intrigue à laquelle il procède, Collé s'efforce de respecter le scénario et l'esprit du conte de Crébillon.

Concentration dramatique et «transposition » du texte-source : une fausse suite, centrée sur l'histoire de Néadarné

Le conte de Tanzaï, sur un scénario de double inconstance imposée à des protagonistes désireux de consommer leur union et de vivre totalement leur amour, complique la structure de base du conte en redoublant l'épreuve au masculin puis au féminin, ce qui a pour effet d'allonger la durée de l'histoire ; à cette première modification, s'ajoute un phénomène d'emboîtement, puisque l'histoire des deux personnages principaux est structurellement interdépendante d'une autre histoire : celle de Moustache et du prince Cormoran dont le désenchantement, la délivrance et le bonheur amoureux sont liés à l'action de Néadarné. Si les deux héros progressent et dépassent les obstacles 
grâce à l'intervention du merveilleux, à leur réussite est subordonnée la destinée d'un second couple, lui aussi soumis aux lois du surnaturel.

À partir de l'histoire du conte et en fonction de la scène pour laquelle il écrit, Collé procède à plusieurs aménagements en s'efforçant toutefois de rester fidèle aux grandes lignes de l'histoire de Crébillon. Conformément aux contraintes de la scène privée, Collé opte pour un scénario raccourci et simplifié, à l'action éclair, où ne sont en jeu qu'un nombre réduit de personnages : en 9 scènes totalisant 6 personnages, tout est joué. Sa réécriture obéit au principe de concentration de l'intrigue initiale qu'il réalise par un centrage sur l'aventure de l'héroïne du conte plutôt que sur celle de son héros, ce que suggère la répartition inégale de la présence des personnages dans la pièce : Tanzaï n'est sur scène que durant deux scènes (sc. 2 et sc. 9), largement dominé par Néadarné qui occupe six scènes sur neuf. Cependant, il ne s'agit pas précisément d'une réduction à l'unité du scénario initial, ni de la reprise du seul second volet du conte, mais Collé, tout en remettant en cause la délivrance finale de Néadarné qui clôturait le conte, en imagine une suite qui était inscrite comme un possible dans l'œuvre narrative ${ }^{17}$; son résultat étant nul et non avenu, l'épreuve au féminin est à refaire et à revivre : la rechute de la princesse constitue donc le sujet de l'action. Sa pièce peut alors se situer dans un rapport de continuité temporelle avec le conte : elle commence par une entrevue entre Moustache et Néadarné qui a lieu deux jours après le retour du couple princier dans ses états. Néadarné revenue à Chéchian après la fameuse étreinte libératrice avec Jonquille, le génie séducteur, nantie du secret de Moustache pour faire illusion sur sa virginité, raconte comment elle a abusé du philtre magique au point que « la porte des plaisirs » est à nouveau murée, au grand désespoir de Tanzaï et de la princesse elle-même. Tout l'enjeu de la pièce sera donc de défaire ce blocage.

Le spectateur est donc mis face à un temps ultérieur aux événements de la source narrative, mais au plan de l'action, il est étrangement renvoyé à la situation de la Néadarné du conte qui est comme dupliquée. Collé retrouve donc l'enjeu du conte tout entier, tel qu'il est actualisé dans son second volet, tout en se présentant comme sa suite. Le rapport de continuité temporelle entre la fable de l'hypotexte et celle de la pièce se trouve donc largement biaisé puisque la nouvelle aventure est décalquée sur la première, ce qui autorise à lire la pièce de Collé comme une adaptation du conte de Crébillon.

19 Ce rapport de continuité est d'ailleurs largement souligné par le dramaturge, car sa pièce fonctionne comme une mémoire de sa source. Ses personnages sont dotés d'une histoire, rendue présente dans leurs discours par des références constantes à leur " vécu » antérieur. Dans l'exposition, Moustache et Néadarné rappellent opportunément leurs déboires passés. La princesse désorientée par son nouveau malheur met au compte de personnages absents de la pièce, mais bien présents et actifs dans le conte, sa nouvelle infortune dont elle accuse la fée Concombre. C'est encore Néadarné qui procède, sous la forme du récit, à un rappel de sa malheureuse histoire avec le prince, dans un résumé fulgurant de l'hypotexte :

La nuit de mon hymen, une fée en courroux

Attache une écumoire à mon illustre époux.

Il voyage, il guérit ; à mon tour, je suis nulle ;

L'on m'envoie à Jonquille, un prince sans scrupule,

Qui malgré les raisons que je pus lui donner,

Obtint... d'horreur encor l'on m'en voit frissonner (sc. 1) 

remplit une valeur d'exposition. Il assure ici surtout la relation d'enchaînement entre le conte et la pièce tout en désignant son origine. Dans la même perspective, Saugrenutio égrène ses souvenirs dans la scène 6 devant Néadarné et Moustache :

Je me souviens du jour où votre époux farouche

D'un grand prêtre, aux autels, voulut forcer la bouche

de Collé repose sur une connivence fondée sur un savoir partagé entre le dramaturge et ses spectateurs. Leur mémoire de lecteurs du conte de Crébillon est constamment sollicitée et ravivée par un réseau de références nourri. Ce qui est mis en œuvre, c'est une stratégie de reconnaissance des personnages qui double leur habituelle présentation : le personnage de la scène arrive doté d'une histoire fictive avec laquelle ses nouvelles aventures où l'on retrouve la fée Moustache, le génie Jonquille, et plus curieusement le grand prêtre Saugrenutio sont mises en continuité. Ces liens manifestes et affichés rendent compte de la posture du dramaturge par rapport au conte : il refuse aussi bien la distance critique que la reprise parodique, quel qu'en soit le régime. Thistoire tout en permettant des correspondances et des inversions aux effets comiques. Or Collé, tout en reproduisant finalement le scénario du second volet du conte, y applique cette structure répétitive comme une sorte de principe, à toutes les étapes de son scénario. Il duplique à la fois l'enjeu de l'action, les moyens de son dénouement et le dénouement lui-même. Quels sont les enjeux de cette duplication ?

Collé donne à l'action de sa pièce un double enjeu, ce qui lui permet d'insérer l'histoire incidente de Moustache. Parallèlement au désenchantement de Néadarné, c'est aussi à Moustache que doit bénéficier la réparation du méfait. Cette dernière est en effet particulièrement intéressée à la délivrance de la jeune épousée : ne s'agit-il pas pour elle de se débarrasser cette importune moustache à laquelle elle doit son nom, de voir se détacher cet accessoire qui blesse sa féminité et l'empêche de vivre sereinement ses amours avec Cormoran? Là encore, Collé se glisse dans les pas de Crébillon en exploitant un détail comique du conte.

24 À cette duplication scénarique portant sur l'enjeu de l'action, s'ajoute dans la pièce une duplication des moyens du dénouement : deux voies s'offrent à Néadarné pour réussir enfin son désenchantement : la réitération de l'épreuve avec le génie Jonquille ou encore, solution préconisée par Tanzaï, l'acceptation par le grand-prêtre de l'humiliation consistant à lécher la fameuse écumoire, cette dernière circonstance apparaissant in fine dans le conte avec un autre objectif : celui de prémunir le jeune couple des persécutions à venir de Concombre. Collé concentre sur la seule héroïne le bénéfice de cette action, mais là encore, il tire essentiellement son matériau des éléments fictionnels du conte qu'il intègre dans une autre perspective ou développe habilement; ainsi le libertinage invétéré du grand-prêtre seulement décrit dans le conte est-il mis en scène dans la pièce comme une péripétie, lorsque ce séducteur libertin s'offre à désenchanter Néadarné en se substituant à Jonquille.

Collé use enfin d'une dernière forme de dédoublement, celle du dénouement. Si la fin euphorique est conforme aux canons du conte, elle superpose dans la simultanéité l'action libératrice des deux moyens de la délivrance : au moment même où, sous la menace, le grand-prêtre accepte de lécher l'écumoire, Néadarné cède au Génie 
Jonquille. Ainsi, la levée des deux enchantements est-elle complète et parfaitement aboutie.

Au-delà de la conformité à l'issue heureuse du conte, ce double dénouement s'explique encore par la fidélité au texte source. La scène de l'écumoire, ponctuée de quelques indices merveilleux (un coup de tonnerre et l'envolée de Jonquille sur son nuage), a pour fonction de maintenir Tanzaï dans l'illusion que le désenchantement de Néadarné s'est opéré sans infidélité de sa part. Dans le conte, le niveau d'information des personnages sur l'épisode de la délivrance de Néadarné était également différent. La complicité de Moustache et de Néadarné, d'ailleurs partagée par le lecteur, excluait Tanzaï et l'empêchait d'accéder à la vérité. Parallèlement, il s'agit bien dans la pièce de sauver la vertu de Néadarné aux yeux de l'époux infortuné qui mettra le désenchantement de la princesse au compte de l'acceptation de Saugrenutio et non d'une trahison conjugale.

En outre, la scène de l'écumoire est justifiée par la perspective de la représentation qui impose décence et respect de la bienséance. Nous avons évoqué plus haut l'obstacle que constituait le caractère sexuel de l'action du récit libertin. Face à l'impossibilité de représenter en direct un dénouement de cette nature, Collé trouve une solution qui consiste à doubler la scène interdite, reléguée dans le hors-scène, par une scène visible : s'il est possible de faire voir Saugrenutio léchant l'écumoire, on ne peut qu'indiquer par le recours à un système de signes la réalisation de l'union charnelle de Néadarné et de Jonquille. Si elle a donc pour fonction de maintenir à des niveaux conformes à ceux du conte, la répartition de l'information dans la pièce et de garantir la position des personnages les uns par rapport aux autres ${ }^{18}$, son rôle est d'autre part de signifier que, hors du champ perceptible par le spectateur, l'acte in-visible a eu lieu. L'heureux dénouement sera confirmé par le récit final de Tanzaï qui, dans l'ultime scène, vient témoigner de la réalisation de deux scènes interdites, reléguées dans un espace extérieur à la scène : il rapporte publiquement l'essentiel de la narration du rêve libérateur que lui a fait Néadarné pour expliquer son désenchantement et la réussite de son union charnelle avec son épouse.

Le traitement théâtral de l'intrigue en fausse suite comme la reproduction érigée en principe de la duplication initiale à toutes les étapes de l'action, indique la complexité du rapport au texte source. Bien qu'elle se présente comme un épilogue, la pièce de Charles Collé relève d'un cas particulier de "transformation " pour reprendre le vocabulaire de Genette, et du régime de la " transposition sérieuse », c'est-à-dire sans intention ludique ou satirique. C'est dans une grande proximité et une familiarité avec sa source que le dramaturge a travaillé et qu'il a cherché des solutions aux impossibilités scéniques que posait l'audace de l'œuvre de Crébillon, sans toutefois le trahir : preuve que si le théâtre de société jouit marginalement d'une grande liberté, celui du Duc d'Orléans imposait une réserve de bon aloi, contrainte à laquelle l'adaptateur se soumet. Tout indique que la pièce est faite pour être lue, vue et goûtée par référence à son hypotexte, plus que de manière autonome ou distanciée. Il ne s'agit en aucun cas de s'affranchir d'une source désignée comme le lieu d'origine de la fiction, mais de la revendiquer en s'inscrivant dans son sillage.

Les justifications du choix générique : la transposition de la mixité du conte On l'a dit, les adaptateurs de contes pour la scène choisissent très massivement la comédie et ses avatars comme genre d'arrivée, ce qui est notamment justifié par les scènes pour lesquelles ils écrivent et par le caractère léger de divertissement mondain 
qu'est le conte. Genre mineur au dénouement heureux débouchant parfois sur une morale souriante, le conte s'accommode aisément d'une transposition dans les genres scéniques les plus divertissants. Or, Collé opte pour un genre qui peut surprendre et qui n'est d'ailleurs pas définitivement fixé au moment de l'écriture de la pièce ; un extrait de son journal d'octobre 1754 est significatif à cet égard : « J'ai voulu mettre Tanzaï en tragédie ", écrit-il alors ; mais ultérieurement, il nomme cette même pièce (dans une note rédigée en 1780) " une tragédie badine », et lorsque l'œuvre est insérée dans ses Euvres complètes, elle y figure sous la catégorie de la tragi-comédie. Ces variations indiquent autant la difficulté du dramaturge à trouver un correspondant qui réponde à l'hybridité qu'il lit dans le conte qu'à sa conception d'un genre auquel il recourt en raison de sa plasticité : la tragi-comédie.

Il n'est pas inutile s'interroger en première instance sur les caractéristiques de la tragicomédie. Loin d'être à la mode au milieu du XVIII ${ }^{e}$ siècle, la tragi-comédie avait connu son heure de gloire au XVII ${ }^{\mathrm{e}}$ siècle, au cours d'une période bien délimitée, de 1628 à 1634. Dans son état primitif, ce genre ennemi de la régularité classique et qui « ouvre l'espace d'une modernité ", répond cependant à des normes, notamment définies par Hélène Baby : « la tragi-comédie est une forme dramatique découpée en actes et en scènes, versifiée en alexandrins, dont l'intrigue fictionnelle, susceptible de se dérouler sur plusieurs années et dans plusieurs pays, et constituée par les obstacles que doit traverser un couple de jeunes gens issus de la haute noblesse, se termine par leur heureuse union ${ }^{19}$ ». Toujours selon Hélène Baby, l'action de la tragi-comédie, tout comme son personnel, sont caractérisés par la mixité : un sujet léger, "réduit à l'accomplissement d'une relation sentimentale » est traité dans une action faite de violences et de grands périls qui favorise le mélange social du personnel dramatique. En outre l'action est le plus souvent éclatée, au sens où elle se fonde sur un empilement d'obstacles sans lien de subordination.

31 La réduction radicale de la longueur de l'intrigue à la longueur d'un acte par Collé rend cette définition canonique du genre et en particulier les traits relatifs à l'espace et au temps, assez caducs : dans la pièce de Collé l'espace est unique ("La scène est à Chéchian, dans le palais de Tanzaï ", indique une didascalie initiale) et le temps particulièrement concentré. En ce qui concerne l'action, les obstacles rocambolesques auxquels sont confrontés les héros de la tragi-comédie ne sont pas non plus de mise pour une si courte pièce ; retenons avant tout le critère de la mixité que Collé a reconnue dans le narré et la narration du conte et qu'il a tentée de reproduire à sa manière.

Du côté des personnages, la qualité sociale élevée des deux héros appelait sans hésitation le recours sinon au grand genre de la tragédie, du moins à celui de la tragicomédie, seuls capables de représenter leur rang et leur dignité. Mais si on ne peut pas dire que leurs aventures les confrontent à des roturiers ou à des personnages de basse extraction, le conte met les deux protagonistes en présence de personnages-fées et de génies. La mixité sociale se trouve donc relayée par une hybridité d'un autre ordre, fondée sur la coexistence entre naturel et surnaturel.

Dans la pièce, ce mélange existe, mais il n'est toutefois guère exploité, puisque l'action de la fée Moustache ne relève plus de ses prérogatives magiques mais de la ruse. Collé évacue de l'action le merveilleux dépendant du personnage pour le faire rentrer dans un des rôles types de la tragédie : ainsi Moustache remplit-elle le rôle de confidente et de conseillère dès la scène initiale qui est réglée selon les normes de la scène 
d'exposition du genre dramatique. Un dialogue intime où le problème de Néadarné peut s'exprimer s'instaure. Le rôle de la fée victime, bien que simplifié (elle n'est plus donatrice), n'est donc pas éloigné de celui qu'elle remplit dans le conte où elle est dotée de la parole bienveillante d'une auxiliaire. Dans la pièce, à l'instar des soubrettes de comédie, elle fait également office d'adjuvante à la tromperie dont est victime Tanzaï. Quand au génie Jonquille, conformément à son homologue du conte, il n'use en rien de son pouvoir magique dans ses relations avec Néadarné, mais d'une argumentation fondée sur l'apologie du plaisir; il se recommande "d'un siècle philosophe " pour fustiger la vertu qu'il rapporte au " confus amas de préjugés bourgeois » (sc. 4) : seuls son arrivée et son départ se signalent par le recours au merveilleux qui se trouve réduit au pur spectaculaire.

Au-delà du mélange des personnages qui impose une mixité du genre théâtral, le sujet du conte crée une première disconvenance de ton avec la qualité des personnages ; par rapport au roman sentimental, le conte libertin non seulement transpose l'accomplissement d'une relation sentimentale dans le domaine sexuel, mais il traite la relation amoureuse charnelle au moyen d'un imaginaire et d'aventures comiques : c'est donc par le recours à la comédie que ce sujet peut-être représenté ou évoqué. Mais le conte va plus loin dans la subversion en centrant sa fable sur la sexualité de souverains, en en faisant des anti-héros uniquement préoccupés par une question bien " prosaïque ». Il y a donc dès le texte source, une tension entre l'identité des héros et leur dégradation humoristique dont Collé se souviendra et qui justifie l'entrechoc de deux tons.

Si l'on centre l'analyse sur les grandes étapes de l'action du conte, une seconde disconvenance est perceptible : un premier dénouement par un personnage extérieur s'avérant impossible, - c'est l'épisode Saugrenutio et son refus de lécher l'écumoire dans la première partie du conte -, il sera nécessaire que le couple princier mette luimême la main à la pâte et œuvre activement à sa propre délivrance. Ni l'enjeu de l'action, ni l'action elle-même n'engagent des qualités proprement héroïques ou conformes à la dignité de leur rang. L'aide reçue par chaque personnage de premier plan, celle de Barbacela pour Tanzaï, celle de la fée Moustache pour Néadarné est déterminante dans la domination des épreuves. Leur parcours linéaire parallèle qui conduit au retour attendu à la norme, n'est cependant pas dénué d'obstacles qui se présentent sous la forme de réticences morales des personnages principaux. Les retardements à la poursuite de l'action sont d'ordre psychologique, ils relèvent d'un conflit qui semble insoluble entre les exigences du cœur et celles du désir.

Or, selon Hélène Baby, la tragi-comédie s'appuie sur des obstacles extérieurs « et non sur les conflits de l'âme humaine ", à l'instar de la tragédie. Si l'action menée par les protagonistes du conte n'a rien de noble, si elle débouche sur la dérision, les réticences qu'ils manifestent relèvent bien d'un conflit intérieur : si on se limite à l'exemple de Néadarné, c'est bien le refus de l'infidélité, la volonté d'accorder ses sentiments à sa conduite et de faire de l'union des corps l'aboutissement naturel du sentiment de l'amour, qui l'arrête à plusieurs reprises. Néadarné méconnaît naïvement la puissance du désir et sa capacité à assujettir la volonté. Nul doute que Collé ne reconnaisse dans cette circonstance l'une des caractéristiques propres à la tragédie, ce qui explique que ce classement générique vienne spontanément sous sa plume au moment où il écrit son adaptation. Par sa spécificité de conflit intérieur, l'obstacle s'apparente à celui auquel se heurtent les plus grandes héroïnes tragiques, déchirées entre leur passion et leur 
devoir. Il ne doit rien à l'obstacle extérieur de la tragi-comédie. Dès la scène 1 , la Néadarné de Collé, conformément au personnage source, exprime fortement son dilemme d'épouse vertueuse :

Il me faut donc encore manquer à mon époux ?

[...]

L'amour et l'amitié contre moi tout conspire

[...]

J'adore Tanzaï, Jonquille est mon vainqueur ;

[...]

Mais ma vertu me parle en faveur d'un époux.

Elle formule aussi des remords sur sa faute passée :

Néadarné d'abord ne s'est que trop prêtée

À la nécessité d'être désenchantée ;

J'y pliais ma pudeur, quand du charme détruit

Je crus que Tanzaï recueillerait le fruit.

Cependant comme dans le conte, ce problème de conscience largement développé est résolu avec humour, par une victoire du corps désirant sur les sentiments d'amour et de vertu, qui place la badinerie au-dessus du tragique.

Les hésitations de Collé dans la dénomination de sa pièce s'expliquent par son dessein de fidélité à un conte au métissage particulièrement complexe que la tragi-comédie dans les formes ne pouvait transposer que partiellement. Si la mixité du personnel du genre canonique correspond, à condition de la déplacer, à celle de l'hypotexte, c'est du côté de la tragédie qu'il est conduit par la nature de l'obstacle auquel son personnage principal est confronté. La tragi-comédie est donc davantage pour lui, un mélange du genre tragique et du genre comique, et le seul genre qui autorise à «traiter comiquement les rois et les héros ${ }^{20}$ ", selon la définition du Dictionnaire dramatique. C'est bien là l'une des postures du conte qui multiplie les décalages générateurs d'humour : entre l'identité des héros et la nature du sujet, entre leur qualité sociale et l'action conduite par eux, entre l'expression de leur conflit intérieur et les moyens employés pour le surmonter.

Les conséquences du choix générique : vers la caricature

Si le genre mixte semble particulièrement approprié à la double postulation du conte de Crébillon, c'est-à-dire au mélange du sérieux et du comique, sa mise en œuvre débouche sur une pièce caricaturale où le merveilleux est réduit au pur spectaculaire, où les personnages ne sont plus que des décalques d'eux-mêmes, et où le libertinage devient outrancier. Une des premières raisons de cet effet involontaire est à chercher dans la transformation du style. Si l'on met de côté le pastiche de Marivaux prêté à la fée Moustache, les protagonistes du conte s'expriment dans un langage codifié, marqué au coin du bon goût mondain. Pour peu que la situation devienne difficile, leurs accents prennent aisément pour quelques phrases un ton et un tour dramatiques, marqués par une grandiloquence moquée par le narrateur. Loin de leur conserver un langage naturel en prose, Collé transpose le langage galant en langage dramatique : le grand genre de la tragédie, comme la tragi-comédie imposaient en effet l'usage de l'alexandrin. Certes, les rencontres entre le style sublime et le langage galant ne sont pas inexistantes et certaines métaphores du domaine amoureux ou l'usage d'un vocabulaire moral abstrait se retrouvent de part et d'autre. En outre, ce choix de faire parler les personnages en alexandrins s'accorde en effet à la qualité élevée des héros princiers en leur conférant la dignité de leur rang. Mais il faut bien avouer que l'usage 
de l'alexandrin et dans une moindre mesure, la tentative de Collé pour transposer la voix du récitant, produisent des effets de sens pour le moins inattendus.

Associé au langage noble de la tragédie, l'alexandrin est censé exalter les valeurs aristocratiques et héroïques, et impliquer une éthique, une grandeur d'âme qui définit le héros aux prises avec les passions. Or, dans l'expression sublime de l'amour et de ses souffrances, comme dans la facture du vers, Charles Collé est passé maître : il recourt avec aisance aux images de la fatalité persécutrice, aux métaphores gravitant autour de la flamme, aux hyperboles par le sens ou par la pluralité ou encore à l'usage de l'abstraction. Ce langage détonne cependant avec le réalisme, voire le prosaïsme du problème posé par la pièce. En effet, élever Néadarné au rang d'une héroïne tragique se lamentant sur sa castration, et exprimant en mesure et en termes choisis son conflit intérieur entre la fidélité due à son époux et la tromperie imposée par la nécessité du désenchantement, son déchirement entre l'amour et le désir, et finalement sans transition sa tentation pour deux hommes, n'est pas sans conséquence. C'est que l'alexandrin et le langage de l'excès qu'il impose fige les personnages dans leur position, et accentue entre eux une distance ${ }^{21}$, que viennent souligner les multiples " Madame » dont Néadarné est créditée par Moustache ou Tanzaï. Là où le conte avançait subtilement, nous faisait comprendre les tergiversations, les atermoiements et l'évolution progressive d'une conscience désemparée, jusqu'à susciter la sympathie amusée du lecteur, la pièce travaille en force en faisant passer l'héroïne d'une posture à l'autre sans transition. Le personnage, devenu une marionnette, perd surtout en crédibilité et en vérité. Que penser de la délicatesse de l'héroïne, qui, après avoir refusé les avances du grand-prêtre, court brusquement retrouver Jonquille en se justifiant ainsi :

Au bosquet je vais trouver Jonquille

Oui, j'y vole. Et si c'est manquer à mon devoir,

C'est, tu le vois, Moustache, un coup de désespoir (sc. 6)

Une telle mauvaise foi si abruptement formulée n'a aucune commune mesure avec les longs débats intérieurs qui animent la Néadarné du conte : ces derniers font percevoir, derrière le regard amusé du narrateur, une question intime, qui loin d'être de pure forme, acquiert vérité et profondeur.

Pis encore, le traitement théâtral du mensonge sur sa vertu que Néadarné fait à Tanzaï devant le témoin qu'est Moustache, lorsqu'elle prétend être sortie vierge de son entrevue avec Jonquille :

Mais ma délicatesse,

$\mathrm{Au}$ seul plaisir du cœur réduisant ma tendresse,

Et rebutant Jonquille et ses soins indécents,

A renoncé, pour vous, à vos plaisirs des sens (sc. 2)

Dans ce passage, Collé a voulu réintroduire un point de vue extérieur évaluatif sur le couple princier, en effet constamment présent dans le conte grâce notamment au narrateur. Dans la pièce, ce rôle est assuré par Moustache qui réagit en aparté (c'est-àdire pour le public) en se gaussant avec ironie de cette prétendue vertu : "Quelle rare vertu!» s'exclame-t-elle alors que Tanzaï assure la continuité du dialogue par une reprise involontaire sur le mot que souligne l'accent du désespoir : "Quelle vertu cruelle ! / J'ai seul, peut-être, au monde, une femme fidèle ; ». 

est centrale. Ce « mauvais théologien, séduisant auprès des femmes ", petit-maître à bonnes fortunes, mais qui ne nous était jamais représenté en action, est campé dans la pièce en séducteur impénitent : le type du libertin, "séducteur adoré de cent grâces crédules » selon Moustache, se propose non pas de lécher l'écumoire pour délivrer Néadarné, mais bien plutôt d'en agir avec elle avec les mêmes moyens que Jonquille. Éconduit par Néadarné, il tente de soudoyer Moustache et son langage tourne alors à la grivoiserie : «Et chez moi j'ose croire, / Qu'elle trouvera moins, mais mieux que l'Écumoire » (sc. 7). Cette évolution du personnage doit tout à Collé, mais sa réflexion est une reprise en écho de pensées qui traversaient l'innocente Néadarné à la vue de la fameuse écumoire, à la fin de la nuit de noces ratée. L'infortunée jeune mariée se demandait «si ce que le prince avait perdu, valait, quoi qu'il en dît, ce qu'il venait d'acquérir ${ }^{24}$ ». Cette évaluation du membre viril comparé à l'écumoire relève de la plus pure tradition gauloise et n'était guère décente, même dans le monologue intérieur d'une jeune épousée. Mais son outrance relève ici de deux transpositions : d'une part une pensée trouve son équivalent dans une parole : extériorisée, partagée, elle peut devenir un sujet de discussion. D'autre part, son transfert d'un esprit féminin à une parole masculine la dote d'une obscénité vantarde.

Collé ne procède qu'à une seule invention qui concerne l'action de la pièce : il insère une péripétie destinée à convaincre Saugrenutio de lécher l'écumoire. Cette péripétie 
oriente la pièce vers une grivoiserie à l'orientale, puisque qu'elle place sur la scène le grand-prêtre en victime d'un chantage : il se voit menacé de castration par Azort, le chef des eunuques, seul personnage totalement inventé par Collé, s'il ne cède pas au rituel de l'écumoire. À la scène 8, Saugrenutio, dans un langage emprunté au héros tragique, met fin à son dilemme en choisissant plutôt que la mutilation, d'être nommé patriarche et de régner en maître sur un harem :

La mort n'était rien à mes yeux ;

Mais vivre, pour traîner son opprobre en tous lieux !

Eh ! Comment reparaître, après, devant des femmes ?

J'eusse bravé le fer, le poison et les flammes...

Ils m'ont pris par mon faible; et je lèche.

51 Rien ne manque à cette parodie du langage tragique du héros : ni l'ombre de la mort, ni la bravoure face aux périls. La chute du vers souligne la disconvenance entre le langage et la réalité triviale de l'accommodement. De manière générale, les effets de discordance qui naissent de l'entrechoc de deux vocabulaires, l'un élevé et l'autre bas, au sein même du vers, accentuent la charge comique qui tourne en ridicule le personnage. Ce procédé participe à la satire religieuse que Collé reprend à son compte. Le caractère et l'action du grand-prêtre dans la pièce prolongent en effet la satire du haut clergé, en insistant sur sa lubricité d'ecclésiastique jouisseur qui place au premier plan les plaisirs de la chair.

52 Enfin, si les nombreux dialogues relatifs à la philosophie de l'amour selon Crébillon pouvaient sembler une forme privilégiée pour la scène, force est de constater que la transposition de ceux qui ont été retenus tourne le plus souvent à la caricature. Ainsi la conversation sinueuse et galante sur l'inconstance qui occupait l'un des entretiens entre Moustache et Néadarné dans le conte, traitée par la démesure, se voit associée à l'impudence la plus flagrante. Et lorsque Moustache justifie l'inconstance, comme l'on pouvait s'y attendre, c'est pour prodiguer à Néadarné une véritable leçon de libertinage :

On peut en aimer trois,

Madame, et, quelquefois, a-t-on bien à combattre,

Pour s'en tenir à trois, et n'en pas aimer quatre.

La confidente et conseillère de la princesse, en comptabilisant le nombre des amants potentiels se fonde certes sur les exigences de la nature, mais elle tient crûment des propos qui débouchent sur un comique de l'excès.

Les personnages attachants du conte ont perdu, au-delà de la douce ironie émanée du narrateur et de points de vue internes, leur capacité à émouvoir, tant leur naïveté et leur innocence se sont muées en conscience cynique.

Avec cette pièce, Charles Collé accouche d'« un étrange monstre " qui défie les tentatives de définition. On n'est ni dans l'héroï-comique, ni dans le burlesque, au sens propre, mais dans un genre mixte, où sont associés ton élevé et fond grivois, où les personnages oscillent entre grandeur et vulgarité dans une sorte de grand écart où ils perdent toute vérité. L'auteur était lui-même conscient des effets pervers de ce contraste. Son journal témoigne de son insatisfaction face à cette pièce restée en portefeuille, mais qui avait cependant suscité la convoitise du duc de Clermont en mars 1755 : «J'en suis venu à bout, mais comment? Je n'en suis point content ; et quoique je me prépare encore à la limer et à y travailler beaucoup, je désespère d'en pouvoir rien faire dont je sois satisfait. Je commence à voir que ce sujet n'est pas 
théâtral, mais c'est s'en apercevoir quand on est à terre ${ }^{25}$ ». La réflexion est profonde : le conte de Crébillon par son hybridité générique, par la multiplicité de ses points de vue, par sa dimension parodique, par ses dialogues tout en nuances, bref par sa subtilité narrative était sans doute un hypotexte particulièrement inadéquat à une mise en dialogues pour la scène, sauf à accepter d'emblée une importante perte de substance ou une dérive de son sens. Peut-être cette difficulté tient-elle aussi à la nature même du théâtre qui est voué à montrer, à extérioriser, et qui peine à exprimer notamment l'ambiguïté caractéristique de l'écriture de Crébillon. Ce dernier avait lu cette pièce. Collé assure qu'elle lui "plaisait beaucoup » avant d'ajouter : "Ce juge difficile et caustique m'a toujours assuré que c'était la mieux écrite de mes pièces, et que je n'avais jamais fait de meilleurs vers. En supposant qu'il eût raison, c'est la moindre partie d'une pièce de théâtre que le style ${ }^{26}$ ». Charles Collé n'est pas dupe. Il a bien compris que Crébillon s'était abrité derrière la célébration de son style pour éviter à son ami une déception, et pour s'épargner une louange insincère.

\section{NOTES}

1. Voir Jean Sgard, Crébillon fils, le Libertin moraliste, Desjonquères, 2002, p. 51.

2. Charles Palissot, Nécrologe, p. 3, cité par J. Sgard, ibid., p. 51.

3. Au cours de l'été 1732 , Crébillon joua le rôle de l'ambassadeur de Sardis aux côtés de $\mathrm{M}^{\text {lle }}$ Quinault (dans le rôle de Merto) et de $\mathrm{M}^{\text {lle }}$ Gaussin (qui jouait Carite) dans la comédie des Abdérites, composée par Moncrif (musique de Mouret, ballet composé par le marquis de Clermont d'Amboise et par le marquis de Laugeron) pour le théâtre de société de la duchesse de Brancas (Correspondance dramatique de De Coudray, t. 2, 1778, $3^{\text {e }}$ partie, lettre 5, Annales du théâtre français, p. 65). Collé rapporte dans son Journal, en mars et en avril 1757, la participation de Crébillon à plusieurs rôles dans des pièces jouées chez $\mathrm{M}^{\text {me }}$ de Meaux, dont celui du commandeur dans La Veuve Philosophe et celui de Bartholin dans Nicaise (Journal et Mémoires, Paris, Firmin Didot, 1868, t. 2, p. 80 et p. 87).

4. Crébillon fils, le libertin moraliste, ouvr. cité, p. 58.

5. On peut s'interroger sur les motifs de cette surprenante ou prudente éviction. Une première explication est peut-être à chercher du côté de la psychologie familiale : le domaine du théâtre et du théâtre noble étant d'abord l'apanage du grand tragique qu'est le père, comment ne pas craindre de figurer en singe burlesque du grand homme lorsque l'on choisit la comédie ? D'autre part, dans le strict domaine de la comédie sentimentale et psychologique, Crébillon fils aurait eu à affronter un autre père spirituel, Marivaux, dont la production occupe les deux principales scènes au moment où il fait ses débuts dans la carrière d'homme de lettres. À la fois émule et disciple émancipé du maître dans le domaine de l'écriture narrative, Crébillon préfèrera se garder de croiser encore le fer avec lui en tant qu'auteur de théâtre.

6. Donnée à partir du 11 septembre 1730 avec un succès ininterrompu pendant 34 jours, La Sylphide, comédie à la rédaction de laquelle Crébillon a sans doute collaboré, n'est nullement une adaptation fidèle de la fine rêverie de celui qui, prudemment anonyme, 
fait ses premiers pas dans la carrière. Soumise aux lois du divertissement à l'italienne, traversée de bouffonneries, de soufflets et de coups de bâtons, de contrastes schématiques, de danses et de jeux de scènes spectaculaires, la pièce possède un scénario qui lui est propre : elle met en scène Éraste, le maître, flanqué de son valet Arlequin, suscitant la passion de deux esprits féminins : une sylphide et une gnomide. Mais que de questions communes avec le récit de Crébillon abordées sous le voile de la farce : le pouvoir de pénétration des esprits, la spiritualité de l'amour et son versant charnel, la capacité des esprits à s'incarner...

7. Clément et Laporte, Anecdotes dramatiques, Paris, Duchesne, 1775, t. 1, p. 227. Un prince dont la jeune épouse est enlevée le soir de leurs noces est contraint de l'aller chercher dans une île déserte pour l'extirper des bras d'un génie habile séducteur nommé Gris de lin, derrière lequel il est aisé de reconnaître le génie Jonquille

8. Tanzaï et Néadarné, tragi-comédie en 1 acte, en vers, précédée de La Lecture, Prologue, en prose dans Théâtre de société de Collé. Cette pièce a été réimprimée au XIX ${ }^{\mathrm{e}}$ siècle chez Michel Lévy frères, 1863, dans le tome XXV du Théâtre contemporain illustré, $621^{\mathrm{e}}$ et $622^{\mathrm{e}}$ livraisons sous le titre L'Écumoire, ou Tanzaï et Néadarné, drame en 1 acte.

9. Lettre de Paris du 13 février 1736 évoquant l'année précédente, citée par J. Sgard, CEuvres complètes de Claude Crébillon, Classiques Garnier, 1999, t. 1, p. 606 (désormais OC). 10. D'après le Journal de Collé (ouvr. cité, t. 1, p. 435), il l'aurait rédigée à grand peine en septembre-octobre 1754, soit entre la parution des Heureux Orphelins et le début de celle d'Ah quel conte!

11. Gérard Genette, Palimpsestes, Seuil, 1982, p. 323-327 et p. 330-331.

12. Catherine Ramond, «Théâtralité et merveilleux dans le conte de la première moitié du XVIII ${ }^{e}$ siècle » dans Le Conte merveilleux au XVIII siècle, une poétique expérimentale ? Kimé, 2002, p. 342-352.

13. Ibid., p. 347.

14. Pour un accès à ce corpus où l'on rencontre des auteurs comme Charles Collé, les Grandval père et fils, Piron... voir le recueil Théâtre érotique français au XvIII siècle, JeanJacques Pauvert et Terrain vague, Paris, 1993.

15. Quelques exemples : Acajou et Zirphile de Duclos est adapté en opéra-comique pour la Foire Saint-Germain l'année même de sa publication (1744), un conte tiré du second volume des Sultanes de Guzarate de Gueullette devient sous la plume de Boissy un opéracomique en un acte en 1735, sous le titre Margéon et Katifé. Favart et l'abbé de Voisenon adaptent le conte en vers de Voltaire Ce qui plaît aux dames (1764) dans une comédie, $L a$ Fée Urgèle, destinée au Théâtre-Italien. Fleur d'Épine de Voisenon, comédie en deux actes s'inspire du conte d'Hamilton. Il conviendrait d'établir un inventaire de ces adaptations revendiquées ou non dans leur titre pour étudier ce phénomène de réécriture spécifique qu'est la dramatisation du conte au XVIII ${ }^{\mathrm{e}}$ siècle.

16. Catherine Ramond, art. cité, p. 349.

17. « Néadarné avertit Moustache qu'elle croyait avoir trop répété le secret, et cette généreuse fée, on ne sait comment, y mit ordre » (OC, t. 1, p. 436).

18. Comme dans le conte le lecteur, le spectateur demeure omniscient.

19. Hélène Baby, «Littérarité et généricité : l'exemple de la tragi-comédie en France au XVIII ${ }^{\mathrm{e}}$ siècle », document électronique, Loxias (Loxias 8 Émergence et hybridation des genres).

20. La Porte et Chamfort, Dictionnaire dramatique, t. 3, Paris, chez Lacombe, 1776, p. 308.

21. "La vérité de l'alexandrin [...] est dans sa distance ", écrit Roland Barthes dans une note de son Sur Racine, Seuil, 1963, p. 138. 
22. OC, p. 426.

23. Jacques Schérer, La Dramaturgie classique en France, Nizet, 1986, p. 394 et suiv.

24. OC, p. 307.

25. Journal et Mémoires, ouvr. cité, octobre 1754, p. 434-435. Collé affirme l'avoir alors conservée pour son protecteur, le duc d'Orléans.

26. Ibid., note écrite en 1780.

\section{RÉSUMÉS}

Lorsqu'il tente d'adapter pour le théâtre de société du duc d'Orléans, Tanzaï et Néadarné, Charles Collé entend bien produire une transposition fidèle, sinon à la lettre du moins à l'esprit du conte de Crébillon. La concentration de l'intrigue en une fausse suite, son attention à reproduire la duplication structurelle initiale en témoignent. Afin de traduire la mixité du conte entre sérieux et comique, il opte pour le genre de la tragi-comédie. Mais il tombe dans le travers de l'excès qui outre le libertinage et les effets de contrepoint, et caricature les personnages.

The theatrical adaptation of Tanzaï and Néadarné by Charles Collé : a unintentional caricature of the tale.

When he tries to adapt Tanzaï and Néadarné for the Duc d'Orléans'society theatre, Charles Collé wants to produce a faithful transposition of Crébillon's tale. The reduction of the plot in a false sequel and his wish to copy the initial structural duplication are signs of his fidelity. Charles Collé chooses tragi-comedy to translate the coexistence of seriousness and comedy. Unfortunately this path leads him to excesses in licentiousness and counterpoint effects, and to the caricature of the characters.

\section{AUTEUR}

\section{RÉGINE JOMAND-BAUDRY}

Université de Lyon 3 